\title{
Impact of transcutaneous electrical nerve stimulation on sleep in chronic low back pain: a real-world retrospective cohort study
}

This article was published in the following Dove Medical Press journal: Journal of Pain Research

\author{
Shai N Gozani \\ Thomas C Ferree \\ Martin Moynihan \\ Xuan Kong
}

NeuroMetrix, Inc., Waltham, MA 0245I, USA
Correspondence: Shai N Gozani NeuroMetrix, Inc., 1000 Winter St., Waltham, MA 0245I, USA

Tel + I 78I 3142789

Fax +I 78I 890 I556

Email gozani@neurometrix.com
Objective: The purpose of this study was to determine if transcutaneous electrical nerve stimulation (TENS) improves sleep in chronic low back pain (CLBP).

Background: There is uncertainty over the effectiveness of TENS in CLBP. In most studies, pain intensity has been the primary outcome measure. Although sleep abnormalities are common in CLBP, sleep outcomes have not been evaluated in most studies of TENS effectiveness. Subjective and objective sleep measures are often inconsistent in CLBP, suggesting that perception of sleep and actual sleep may differ.

Methods: This retrospective cohort study evaluated TENS for CLBP over 10 weeks. The source database included demographics, pain characteristics, pain intensity and interference on an 11-point numerical rating scale, adherence and actigraphic sleep data from real-world TENS users. Key inclusion criteria were CLBP with self-reported history of back injury and baseline pain interference with sleep $\geq 4$. Study participants were stratified into improved and unimproved groups based on changes in pain interference with sleep (improved $\geq 1$-point decrease). Actigraphic sleep metrics were compared between the two groups for weeks 1-2 and weeks 9-10.

Results: The inclusion criteria were met by 554 TENS users. There were $282(50.9 \%)$ participants in the improved group and $272(49.1 \%)$ in the unimproved group. The two groups had similar baseline characteristics and high TENS adherence. At the weeks 1-2 assessment, there were no differences among actigraphic sleep. At the weeks 9-10 assessment, there was a difference in total sleep time, with the improved group sleeping 29 minutes longer. In addition, the periodic leg movement (PLM) index was lower in the improved group.

Conclusion: Regular TENS improved self-reported and objective sleep measures in individuals with CLBP. When compared to the unimproved group, the improved group had longer total sleep time and fewer PLMs. Sleep may be an important outcome for TENS effectiveness in CLBP.

Keywords: chronic pain, chronic low back pain, transcutaneous electrical nerve stimulation, sleep, actigraphy, periodic leg movements, total sleep time

\section{Introduction}

Transcutaneous electrical nerve stimulation (TENS) is a non-invasive treatment for chronic pain without significant side effects. Conventional TENS is delivered through surface electrodes to generate a strong, nonpainful sensation. The resulting stimulation of large diameter, deep tissue afferents produces pain relief by decreasing central sensitization and enhancing descending pain inhibition. ${ }^{1}$ TENS has been used for the symptomatic treatment of chronic low back pain (CLBP) since the $1970 \mathrm{~s}^{2}$ A recent meta-analysis concluded that TENS was superior to placebo or control in reducing pain intensity in non-specific low back pain; however, the selected studies exhibited 
heterogeneity. ${ }^{3}$ Another recent meta-analysis found that TENS reduced CLBP for treatment periods $<5$ weeks. ${ }^{4}$ A number of other systematic reviews and meta-analyses concluded that TENS was ineffective for CLBP. ${ }^{5-8}$ Several factors may contribute to negative outcomes in TENS clinical trials including inadequate stimulation dose, ${ }^{3,9,10}$ use of effective co-interventions, ${ }^{10}$ poor adherence, ${ }^{10,11}$ short study length, ${ }^{10}$ and insufficient sample size. ${ }^{10}$ Another issue may be the choice of outcome measures. In most TENS studies, pain intensity has been the primary outcome measure. ${ }^{10}$ However, individuals with chronic pain use TENS in various ways to improve their quality of life. ${ }^{12}$ It is possible that the focus on pain intensity has obscured overall TENS clinical utility. The uncertainty over the effectiveness of TENS in CLBP may be limiting its use despite an excellent safety profile and the need for non-pharmacological pain relief options.

More than half of individuals with CLBP suffer from impaired sleep. ${ }^{13-15}$ The abnormalities include short sleep duration, delayed sleep onset, low sleep efficiency, increased awakenings, and altered sleep architecture. ${ }^{13,16-18}$ For these reasons, sleep is a relevant outcome measure of therapeutic effectiveness in CLBP. Moreover, chronic pain patients identify improved sleep as an important therapeutic goal. ${ }^{19,20}$ Several recent studies reported a decrease in self-reported pain interference with sleep following 2 months of TENS use in individuals with multi-site chronic pain. ${ }^{21-23}$ Subjective and objective sleep measures may be discordant in CLBP, suggesting that perception of sleep and actual sleep may differ, and both should be evaluated. ${ }^{16}$ The impact of TENS on objective sleep metrics in chronic pain has not been evaluated. The purpose of this real-world study was to evaluate the hypothesis that TENS users with CLBP will experience improvement in both self-reported and objective measures of sleep. Demonstration of an impact of TENS on both subjective and objective sleep in CLBP will increase confidence in its clinical value.

\section{Methods}

\section{Data source and study population}

The source for this retrospective cohort study was a database that collects real-world adherence and clinical data from individuals self-administering TENS for chronic pain. Data are uploaded to the database via a mobile application linked to the TENS device. The analyses were performed on a copy of the database with data collected between January 2, 2017 and August 29, 2018. The database includes self-reported user demographics, self-reported pain characteristics, pain ratings, objective adherence data, and objective sleep data. ${ }^{22,23}$
Submission of demographic and clinical data to the database via the mobile application is voluntary. In addition, capture of device data (ie, adherence and sleep) requires periodic synchronization of the device with the mobile application. For these reasons, although any individual contributing to the database was eligible for the study, only a subset provided a complete dataset necessary for inclusion.

Individuals meeting the following criteria were included as study participants: 1) age, gender, height, and weight recorded in database; 2) pain duration indicated as $>3$ months, 3) pain frequency indicated as at least several times a week, 4) baseline pain rating recorded in database, 5) baseline pain interference with sleep $\geq 4$ (ie, moderately disturbed sleep interference), ${ }^{24} 6$ ) list of pain sites included low back pain, 7) list of self-reported painful health conditions included prior back injury, 8) 10 weeks of continuous adherence data, starting on first day of device use, recorded in database, and 9) 10-week follow-up pain rating recorded in database. Prior back injury was a requirement to reduce the likelihood of including participants with incidental low back pain. In addition, tissue and nerve injury is associated with central sensitization ${ }^{25}$ which may increase the likelihood of responding to TENS. ${ }^{3}$

All device users electronically consented to the use of their de-identified data for clinical research during the process of establishing a database account. This study was institutional review board exempt because the investigators used a database without personal identifying information (Code of Federal Regulations, Title 45, Department of Health and Human Services, Part 46, Protection of Human Subjects, Section 101(b)(4)). The reporting of this study followed the STROBE recommendations. ${ }^{26}$

\section{Transcutaneous electrical nerve stimulation}

Contributors to the database used the same TENS device $\left(\right.$ Quell $^{\circledR}$, NeuroMetrix Inc., Waltham, MA, USA). ${ }^{21,22}$ Instructional materials were those normally included with commercial purchase; no additional training was provided. The user guide describes device placement, explains the importance of stimulation at a "strong but comfortable" level, and suggests daily use with at least 3 hours of stimulation. The device is placed on the upper calf and comprised a one-channel electrical stimulator, a stretchable band to secure the stimulator to the leg, and an electrode array. ${ }^{21}$ The electrode array comprised four hydrogel pads, each $\sim 15 \mathrm{~cm}^{2}$, configured as two 30 $\mathrm{cm}^{2}$ electrodes. When placed on the upper calf, the electrode array wraps around the leg overlapping sensory dermatomes 
S2 through L4. These dermatomes are typically targeted when treating low back pain with TENS, ${ }^{27}$ although placement is often in the lower back region rather than distally. However, TENS has widespread effects that may obviate the need for co-localization of electrodes and pain..$^{23,28-30}$ The peak output voltage and current are $100 \mathrm{~V}$ and $100 \mathrm{~mA}$, respectively. The stimulation waveform is current regulated, rectangular and biphasic with a pulse duration of $280 \mu \mathrm{s}$. The inter-pulse intervals are pseudo-random with a mean frequency of 80 Hz. Prior to first use, the device is calibrated to the user's sensation threshold by an algorithm using both ascending and descending method of limits. Subsequent stimulation is automatically controlled. The initial therapeutic level is set so that the pulse charge is $5 \mathrm{~dB}$ above sensation. This level is typically perceived as "strong but comfortable". The stimulation intensity is then periodically increased by an adaptive algorithm to compensate for nerve de-sensitization and to activate deep tissue sensory afferents. ${ }^{31}$ The user may also manually decrease or increase the intensity. Therapy sessions are 60 minutes, starting every other hour while the device is worn. Users also have the option of 30-minute sessions and 30-minute inter-session periods.

Several adherence parameters are electronically tracked by the TENS device. Utilization is the percentage of days with a minimum of 30 minutes of stimulation. ${ }^{10}$ It is a measure of how regularly TENS is used. Prior studies have shown a dose-response association between utilization and chronic pain outcomes. ${ }^{22,23,32}$ Sleep utilization is the percentage of nights with at least 30 minutes of stimulation during sleep. Hours/week is the average number of hours of stimulation per week. Sensation threshold is the current, in milliamps, at which electrical stimulation is first perceived. This value is determined by the aforementioned calibration algorithm. Stimulation intensity is the ratio of therapeutic current to the sensation threshold expressed in decibels.

\section{Actigraphy}

Actigraphy is a non-invasive method to assess sleep in ambulatory settings. ${ }^{33}$ Actigraphy devices are worn on the body and continuously record and analyze movements from which sleep metrics are derived under the principle that sleep is characterized by the relative absence of movement. The method has good sensitivity and moderate specificity for detecting sleep when compared to gold-standard polysomnography. ${ }^{34-36}$ Traditional actigraphy cannot resolve sleep stages and therefore does not characterize sleep architecture. Common actigraphic sleep metrics include total sleep time (total time asleep from sleep onset to waking; measured in minutes), sleep efficiency (percentage of total time in bed trying to sleep that is actually spent sleeping; measured as \%), sleep onset latency (time to fall asleep; measured in minutes), and wake after sleep onset (total time awake from sleep onset to waking; measured in minutes). ${ }^{37}$ Consensus recommendations for sufficient sleep duration and acceptable sleep quality have been published by the National Sleep Foundation. ${ }^{37,38}$ Actigraphy can also identify periodic leg movements (PLMs), which are repetitive cramping or jerking of the legs during sleep, ${ }^{39}$ with acceptable accuracy as referenced to polysomnography. ${ }^{34,40,41}$ A PLM index $\geq 5 /$ hour is considered abnormal. ${ }^{39}$ Restless leg syndrome $^{39,42}$ and chronic pain ${ }^{43}$ are both associated with an elevated PLM index. Although PLMs are a marker for pathophysiological processes during sleep, ${ }^{44-46}$ they may not directly disrupt sleep. ${ }^{47,48}$

Most actigraphy devices are worn on the wrist; however, alternative locations such as the leg are valid. ${ }^{33}$ The TENS device used in this study contains a 3-axis MEMS accelerometer for actigraphy measurements. Accelerometer signals are acquired at $50 \mathrm{~Hz}$ and processed to 1-minute epochs. Epochs are classified as sleep if the user is recumbent and the mean activity within the epoch is below a threshold. Following classification of epochs, sleep metrics are determined. For measurement of PLMs, the $50 \mathrm{~Hz}$ data stream is averaged down to $10 \mathrm{~Hz}$ and high pass filtered at $0.5 \mathrm{~Hz}$. Individual leg movements and PLM sequences are automatically detected by applying World Association of Sleep Medicine standards. ${ }^{49}$ Sleep data are stored on the device until the next connection with the mobile application. The actigraphic methods used in this study have been compared to polysomnography in patients referred to an academic sleep laboratory. ${ }^{50,51}$

Actigraphic sleep measurements vary night to night and therefore sleep data should be aggregated over several nights to enhance reliability. ${ }^{52}$ In this study, participant sleep was determined for an initial assessment (weeks 1-2) and a final assessment (weeks 9-10) as shown in Figure 1. The representative sleep metrics for each assessment were the median value among all nights within the 2 -week period with data, subject to a minimum of three nights. ${ }^{52}$ Nights with total sleep time of $<4$ hours or $>10$ hours were excluded because of the likelihood of a data collection error, such as the device separating from the participant. Actigraphic sleep data were typically collected on nights during which participants used their TENS device therapeutically. Therefore, objective sleep outcomes may have reflected the concurrent benefit of TENS on sleep in addition to a cumulative effect. Evaluation of outcomes during TENS use is recommended for maximizing study fidelity. ${ }^{10}$ 


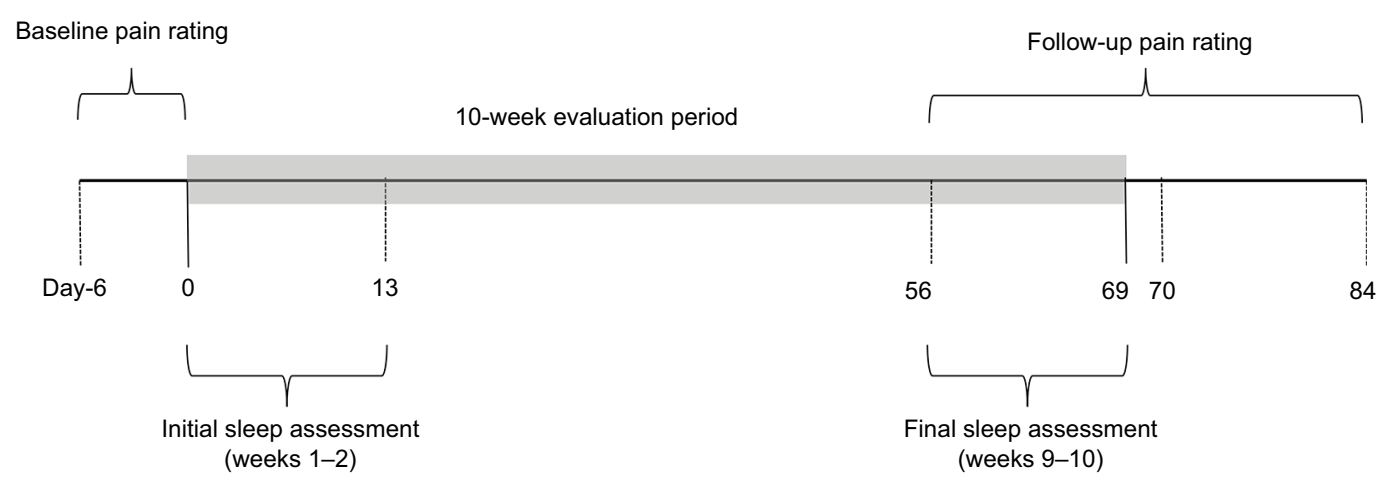

Figure I Schematic illustrating timing of pain ratings, actigraphic sleep assessments and therapy evaluation period for study participants.

\section{Pain ratings and participant stratification}

Pain ratings were electronically entered using the mobile application. Users who opted in to receiving push notifications on their smartphone received regular reminders to rate their pain. However, as a real-world study, the timing and frequency at which users rated their pain could not be controlled. Each pain rating included pain intensity and pain interference evaluated on an 11-point numerical rating scale (NRS) derived from the Brief Pain Inventory - Short Form. ${ }^{53}$ Four pain domains were tracked as follows: average pain intensity over the past 24 hours, pain interference with sleep over the past 24 hours, pain interference with activity over the past 24 hours, and pain interference with mood over the past 24 hours. Pain ratings defined over an NRS are ordinal variables; however, in this study they were analyzed as numerical variables.

Figure 1 shows the definition of the baseline and followup pain ratings. The baseline pain rating occurred on the first day (day 0) of TENS use or within the prior 6 days. The follow-up pain rating occurred between days 56 and 84 (ie, \pm 2 weeks of day 70 ). If more than one pain rating was available within the baseline or follow-up window, the rating closest to day 0 or day 70 , respectively, was used. If more than one pain rating was available on a given day, the first was used. Study participants were stratified into the following two groups; those with improved pain interference with sleep (decrease $\geq 1$ from baseline to follow-up ${ }^{54}$ ) and those with unimproved pain interference with sleep (no change or increase $\geq 1$ from baseline to follow-up).

\section{Statistical analyses}

Device usage, demographics, and pain characteristics were quantified by the mean and SD if numerical variables and by proportions if categorical variables. The statistical significance of group comparisons was evaluated by the two-sample $t$-test with equal variance for numerical variables. Key results were confirmed by the non-parametric two-sample Wilcoxon rank-sum test to verify that statistical significance was not a distribution artefact. The PLM index was log transformed prior to statistical testing to correct for a positive skew. Effect sizes were determined using the Cohen's $d$ measure. The Pearson's chi-squared test was applied to categorical variables (eg, gender) and to contingency tables (eg, pain duration) to evaluate how likely the observed proportions arose by chance. A sensitivity analysis was conducted against changes to inclusion criteria and the definition of improved pain interference with sleep. Analyses were performed with Stata Version 15.1 (StataCorp, College Station, TX, USA).

\section{Results}

A total of 554 TENS users met the inclusion criteria and formed the study group. This cohort was drawn from an initial population of 6,058 individuals who had a complete set of baseline data (criteria 1-4). A first set of 3,610 (59.6\%) individuals were excluded because they did not indicate low back pain (criterion 5) or specify a prior back injury (criterion 6). An additional 649 (10.7\%) individuals were excluded because of a baseline pain interference with sleep $<4$ (criterion 7). Finally, 1,245 (20.6\%) individuals were excluded because they either did not have 10 weeks of adherence data (criterion 8) or a follow-up pain rating (criterion 9) in the database.

There were 282 (50.9\%, 95\% CI: 46.7, 55.1) participants in the improved group and 272 (49.1\%, 95\% CI: 44.9, 53.3) in the unimproved group. The baseline to follow-up evaluation period was $67.0 \pm 5.0$ days for the improved group and $67.8 \pm 5.8$ days for the unimproved group $(P=0.088)$. Table 1 shows baseline demographics and pain characteristics for the two groups. The study participants were generally older 
Table I Comparison of demographics and baseline pain characteristics between study participants with improved and unimproved pain interference with sleep

\begin{tabular}{|c|c|c|c|}
\hline Characteristic & Improved (282) & Unimproved (272) & $P$-value \\
\hline Female gender: $\%$ & 55.3 & 50.7 & 0.280 \\
\hline Age: mean (SD) & $55.2(12.8)$ & $54.4(11.8)$ & 0.443 \\
\hline BMI $\left(\mathrm{kg} / \mathrm{m}^{2}\right):$ mean $(\mathrm{SD})$ & $31.6(7.7)$ & $31.6(7.2)$ & 0.985 \\
\hline Duration of pain (years): $\%$ & & & 0.064 \\
\hline$<1$ & 0.4 & 2.9 & \\
\hline $1-3$ & 15.2 & 11.4 & \\
\hline $4-10$ & 30.5 & 30.9 & \\
\hline$>10$ & 53.9 & 54.8 & \\
\hline No. pain sites: mean (SD) & $5.9(2.5)$ & $6.0(2.4)$ & 0.593 \\
\hline Lower extremity pain: \% & 93.3 & 94.9 & 0.429 \\
\hline Upper extremity pain: \% & 71.6 & 71.3 & 0.936 \\
\hline Neck pain: \% & 61.0 & 61.0 & 0.993 \\
\hline Head pain: \% & 22.7 & 20.6 & 0.547 \\
\hline No. painful health conditions: mean (SD) & $5.7(2.3)$ & $5.8(2.5)$ & 0.458 \\
\hline Pain frequency: \% & & & 0.078 \\
\hline Daily & 96.1 & 98.5 & \\
\hline Weekly & 3.9 & 1.5 & \\
\hline Time of worst pain: $\%$ & & & 0.194 \\
\hline All day & 62.4 & 67.3 & \\
\hline At night or when sleeping & 8.5 & 6.6 & \\
\hline In morning & 11.7 & 6.6 & \\
\hline At rest & 2.8 & 2.2 & \\
\hline With activity & 10.3 & 12.9 & \\
\hline Weather sensitive: \% & 85.5 & 87.5 & 0.532 \\
\hline \multicolumn{4}{|l|}{ Baseline pain: mean (SD) } \\
\hline Pain intensity & $7.1(1.7)$ & $6.9(1.9)$ & 0.390 \\
\hline Pain interference with sleep & $7.4(1.9)$ & $6.4(1.9)$ & 0.000 \\
\hline Pain interference with activity & 7.7 (1.9) & 7.5 (I.9) & 0.236 \\
\hline Pain interference with mood & $7.3(2.2)$ & $7.3(2.1)$ & 0.922 \\
\hline
\end{tabular}

Notes: No. pain sites, number of self-reported pain sites from feet/ankles, legs, hips, lower back, trunk, hands/wrist, arms, shoulders, neck, and head. No. painful health conditions, number of self-reported pain related conditions from arthritis, fibromyalgia, herniated disc, spinal stenosis, prior leg/foot injury, prior back injury, prior arm/hand injury, diabetes, complex regional pain syndrome, shingles, restless leg syndrome, multiple sclerosis, migraine, and cancer. Weather sensitive, \% of participants responding "yes" to the question "Does weather affect your chronic pain?" $P$-value is for two-sample $t$-test with equal variance or Pearson's chi-squared test.

adults, overweight, and equally split between male and female gender. Most participants reported chronic pain of $\geq 4$ years duration that was characterized by every day pain. More than $95 \%$ of participants had moderate $(32.3 \%$, 4-6 points) or severe $(64.3 \%, \geq 7$ points) pain at baseline. Participants reported substantial pain interference with sleep, activity, and mood at baseline. The only statistically significant difference between groups was for pain interference with sleep, which was higher in the improved group (7.4 \pm 1.9 vs $6.4 \pm 1.9, P<0.001)$. Multi-site pain ( $\geq 3$ sites) was reported by $92 \%$ of participants. The study participants had extensive pain-related medical histories, averaging over five painful health conditions. The three most commonly reported were arthritis $(69.7 \%)$, herniated disc $(50.4 \%)$, and prior neck injury (48.7\%). The only statistically significant difference between the groups was for prior neck injury (improved $53.2 \%$ vs unimproved $44.1 \%, P=0.033$ ).
Table 2 shows the change in pain ratings from baseline to follow-up. The overall study population exhibited statistically significant improvement in all pain ratings. The improved group exhibited substantial reductions in pain interference and a moderate decrease in pain intensity. The unimproved group demonstrated worsening of pain intensity and pain interference with sleep and activity, but no change in pain interference with mood.

Table 3 compares adherence in the two groups. The improved group had higher utilization $(77.0 \pm 26.0 \mathrm{vs}$ $70.2 \pm 29.2, P=0.004)$ and a trend toward higher sleep utilization $(39.9 \pm 32.5$ vs $35.1 \pm 31.0, P=0.081)$. There was no difference in weekly TENS use between the groups $(42.0 \pm 23.8$ hours/week vs $39.1 \pm 26.3$ hours/week, $P=0.176)$. There were no statistically significant differences between the two groups for either sensation threshold or stimulation intensity. The stimulation intensity was $\sim 5 \mathrm{~dB}$ in both groups, which 
represents a 1.8-fold ratio between the therapeutic stimulation intensity and the sensation threshold.

Table 4 compares actigraphic sleep metrics in the two groups. Sleep data were available for $58.9 \%$ of the improved group at the initial assessment and $41.8 \%$ at the final assessment. Similarly, sleep data were available for $58.8 \%$ of the unimproved group at the initial assessment and 39.0\% at the final assessment. Sleep metrics were determined from an average of $\sim 8$ (range 3-14) nights of sleep for both groups at both assessments. At the initial assessment (weeks 1-2), there were no statistically significant differences between the groups. Participants in both groups had a mean total sleep time slightly below 7 hours (normal 7-9 hours) and a mean sleep efficiency at the low end of the normal range (normal $>85 \%$ ). Both groups had an abnormal mean PLM index (normal $<5$ /hour). At the final assessment (weeks 9-10), there was a statistically significant difference in total sleep time between the groups, with the improved group sleeping 29 minutes longer than the unimproved group (424 \pm 81 vs $395 \pm 81, P=0.008$ by two-group $t$-test and $P=0.013$ by two-sample Wilcoxon rank-sum test). In addition, the PLM index was lower in the improved group $(6.8 \pm 8.8$ vs $9.3 \pm 12.5, P=0.022$ and $P=0.035$ by two-sample Wilcoxon rank-sum test). The effect sizes were 0.36 (95\% CI: 0.09-0.62) for the total sleep time and 0.32 (95\% CI: $0.05-0.59$ ) for the PLM index.

\section{Sensitivity analysis}

A sensitivity analysis was conducted to explore the impact of modifications to the inclusion criteria and definition of improved pain interference with sleep on the total sleep time and PLM index at the final assessment. The first sensitivity analysis eliminated the requirement for a minimum baseline

Table 2 Change in pain ratings from baseline to follow-up in all study participants and for participants with improved and unimproved pain interference with sleep

\begin{tabular}{|l|l|l|l|l|}
\hline Pain rating: mean (SD) & All participants $\mathbf{( N = 5 5 4 )}$ & Improved (N=282) & Unimproved (N=272) & P-value \\
\hline Pain intensity & $-0.4(2.4)^{*}$ & $-1.6(2.3)^{*}$ & $0.9(1.8)^{*}$ & $<0.00 I$ \\
Pain interference with sleep & $-0.9(2.8)^{*}$ & $-3.0(2.0)^{*}$ & $1.4(1.4)^{*}$ & $<0.001$ \\
Pain interference with activity & $-1.1(2.8)^{*}$ & $-2.5(2.7)^{*}$ & $0.3(2.0)^{* *}$ & $<0.001$ \\
Pain interference with mood & $-1.2(2.8)^{*}$ & $-2.5(2.7)^{*}$ & $0.3(2.2)$ & $<0.001$ \\
\hline
\end{tabular}

Notes: $P$-value is for comparison of improved and unimproved groups by two-sample $t$-test with equal variance. *Indicates that group mean is greater or less than 0 by the one-sample $t$-test with $P<0.00 \mathrm{I}$. **Indicates that group mean is greater or less than 0 by the one-sample $t$-test with $P<0.05$.

Table 3 Comparison of TENS adherence over 10-week evaluation period between study participants with improved and unimproved pain interference with sleep

\begin{tabular}{|l|l|l|l|}
\hline Variable: mean (SD) & Improved (N=282) & Unimproved (N=272) & P-value \\
\hline Utilization (\%) & $77.0(26.0)$ & $70.2(29.2)$ & 0.004 \\
Sleep utilization (\%) & $39.9(32.5)$ & $35.1(31.0)$ & $0.08 \mathrm{I}$ \\
Hours/week & $42.0(23.8)$ & $39.1(26.3)$ & 0.176 \\
Sensation threshold (mA) & $16.5(13.5)$ & $17.8(14.3)$ & 0.268 \\
Stimulation intensity (dB) & $5.3(5.3)$ & $5.1(5.6)$ & 0.732 \\
\hline
\end{tabular}

Notes: $P$-value is for two-sample $t$-test with equal variance. Sleep utilization, $\%$ of nights with at least 30 minutes of stimulation during sleep. Stimulation intensity, ratio of stimulation intensity to sensation threshold expressed in decibels. Utilization, \% of days with at least 30 minutes of stimulation.

Table 4 Comparison of actigraphic sleep metrics between study participants with improved and unimproved pain interference with sleep

\begin{tabular}{|l|l|l|l|l|}
\hline \multicolumn{2}{|l|}{ Weeks I-2 } & \multicolumn{2}{l|}{ Weeks 9-I 0 } \\
\hline Variable: mean (SD) & Improved (N= I66) & Unimproved (N=160) & Improved (N=I I 8) & Unimproved (N= I06) \\
\hline Total sleep time (min) & $408(74)$ & $405(80)$ & $424(8 I)$ & $395(8 I)^{*}$ \\
Sleep efficiency (\%) & $87.7(0.7)$ & $87.8(0.7)$ & $88.3(0.8)$ & $88.0(0.7)$ \\
Sleep onset latency (min) & $30.3(32.6)$ & $28.0(36.8)$ & $32.4(49.3)$ & $25.1(28.9)$ \\
Wake after sleep onset (min) & $27.2(15.7)$ & $28.6(18.1)$ & $26.9(24.0)$ & $28.2(16.9)$ \\
PLM index (PLM/hour) & $8.0(8.8)$ & $8.7(13.7)$ & $6.8(8.8)$ & $9.3(12.5)^{*}$ \\
Recording nights & $8.8(3.6)$ & $8.6(3.4)$ & $8.8(3.4)$ & $8.3(3.6)$ \\
\hline
\end{tabular}

Notes: $* P<0.05$ by two-sample $t$-test with equal variance. PLM index log was transformed prior to statistical testing.

Abbreviation: PLM, periodic leg movement. 
pain interference with sleep of 4 (criterion 5). There was a decrease in the proportion of participants in the improved group $(43.5 \%)$ but minimal impact on the difference in total sleep time between the groups (426 \pm 81 vs $399 \pm 79$, $P=0.005)$. The $P$-value for the group difference in the PLM index $(6.8 \pm 9.0$ vs $8.9 \pm 11.8, P=0.059)$ increased above the predetermined statistical significance level of 0.05 . The second sensitivity analysis eliminated the requirement for 10 weeks of continuous adherence data (criterion 8). Neither the proportion of participants in the improved group (50.6\%) nor the difference in total sleep time between the groups changed ( $425 \pm 81$ vs $395 \pm 81, P=0.006$ ). The $P$-value for the group difference in the PLM index (6.8 \pm 8.7 vs $9.1 \pm 12.4$, $P=0.034$ ) remained under the predetermined statistical significance level of 0.05 . The third sensitivity analysis redefined improved pain interference with sleep as at least a 2-point decrease. As expected, the proportion of participants in the improved group decreased (38.3\%) but there was no change in the total sleep time difference between the groups $(428 \pm 81$ vs $399 \pm 82, P=0.010)$. Statistical significance for the group difference in the PLM index was lost $(7.2 \pm 9.6$ vs $8.5 \pm 11.5$, $P=0.134)$. Similar results were obtained for definitions of $15 \%$ and $30 \%$ relative decrease in pain interference with sleep. Overall, total sleep time appeared robust to modifications of the inclusion criteria and definition of improved pain interference with sleep while the PLM index exhibited sensitivity and should be interpreted with greater caution.

\section{Discussion}

The purpose of this study was to determine if TENS users with CLBP who report a decrease in pain interference with sleep also exhibit improved actigraphic measures of sleep. There were two key findings. The first was that half of the study participants experienced at least a minimal clinically important change in pain interference with sleep over the 10 -week evaluation period. These participants were classified as having improved pain interference with sleep. The improved and unimproved groups had similar demographics and baseline characteristics. Both groups had multi-site pain, moderate to severe pain at baseline, and multiple pain-related conditions. These features are consistent with poor health and multimorbidity associated with CLBP ${ }^{55}$ The only statistically significant difference at baseline was a higher pain interference with sleep in the improved group. A similar dependence on baseline pain interference with sleep was reported for pregabalin in neuropathic pain. ${ }^{24}$

The second key study finding was that the improved group exhibited better sleep by certain actigraphic sleep metrics compared to the unimproved group. Specifically, the improved group had longer total sleep time and a lower PLM index than the unimproved group at the end of the evaluation period (ie, weeks 9 and 10). The difference in total sleep time was not accompanied by differences in sleep efficiency, sleep onset latency, or wake after sleep onset. This pattern suggests that the two groups had similar sleep quality, ${ }^{37}$ but participants in the improved group remained asleep longer. This is an important result as sleep duration predicts both near-term ${ }^{56}$ and long-term ${ }^{57}$ changes in chronic pain. The explanation for the selective impact on sleep duration is unclear but may relate to an influence of TENS on abnormal rest-activity patterns associated with chronic pain as discussed further below. The difference in PLM index between the two groups has a less direct interpretation. There is disagreement on whether PLMs directly disrupt sleep or are primarily markers for disturbed sleep..$^{47,48,58}$ In either case, the lower PLM index in the improved group is suggestive of decreased pathology during sleep. ${ }^{58}$ Adherence variables for study participants were similar to successful long-term users of TENS..$^{59,60}$ Therefore, the results in this study likely represent TENS effectiveness at high adherence.

Self-reported pain and sleep are sensitive to interpretation and influenced by psychological and social factors. An obvious limitation of self-reported sleep is the loss of consciousness during sleep, which makes individuals particularly poor reporters of sleep characteristics. Objective measures are generally insensitive to psychosocial factors and therefore should be more reliable across participants within a study and over time. Self-reports of low sleep duration and poor sleep quality are often not corroborated by actigraphy in chronic pain patients. ${ }^{16,18,61}$ We believe this is the first study to demonstrate that TENS improves an objective outcome measure in a chronic pain cohort. This finding enhances confidence in a mechanistic link between TENS and improved clinical outcomes in patients with CLBP.

The present study suggests that TENS improves subjective and objective measures of sleep; however, the biological mechanisms are unknown. It is likely that a portion of the sleep benefit in the improved group causally followed from decreased pain. ${ }^{62,63}$ TENS activates descending pain inhibition and decreases sensitization of neurons in the ascending pain pathway. ${ }^{1,64}$ This modulation of pain processing may also explain the lower PLM index in the improved group. Although the neurologic generator for PLMs is unknown, evidence points to enhanced spinal cord excitability and a relationship to the spinal flexion reflex ${ }^{65}$ which is closely associated with pain. ${ }^{66,67}$ It is possible TENS reduced both 
pain and PLMs through an overlapping enhancement of central inhibition and reduction in central excitation. Consistent with this hypothesis, several studies have demonstrated that TENS reduces the flexion reflex ${ }^{68,69}$ and spasticity. ${ }^{70-72}$ There is also preliminary evidence that TENS reduces symptoms of restless leg syndrome; ${ }^{73}$ however, that study did not measure PLMs.

It is also possible that TENS directly improved sleep through activation of CNS areas that regulate sleep rather than pain. There are reports of TENS altering sleep patterns in patients with CNS disease. For example, TENS may partially correct rest-activity rhythm abnormalities in patient with Alzheimer's disease. ${ }^{74}$ The study authors hypothesized that TENS activates the hypothalamic suprachiasmatic nuclei, which regulates biological clocks. Abnormal rest-activity patterns have been reported in chronic pain conditions including fibromyalgia, ${ }^{75}$ painful diabetic neuropathy, ${ }^{76}$ and knee osteoarthritis with insomnia. ${ }^{77}$

A contribution from non-specific effects, including regression to the mean and placebo, cannot be excluded as this study was observational. Regression to the mean is unlikely to explain the group difference in total sleep time at the final assessment because both groups had similar sleep metrics at the first assessment. Objective outcomes may be less susceptible to placebo than patient reported outcomes; ${ }^{78}$ however, physiological changes can occur in response to placebo. $^{79}$

\section{Study limitations}

This study had several limitations. First, the study was retrospective which may increase the potential for bias. It may be beneficial to confirm the findings with a prospective design. Second, the impact of co-interventions, such as pain medications, could not be evaluated because this information was not available in the data source. Concurrent analgesic use may have masked or enhanced the apparent therapeutic benefit of TENS. Third, patient reported assessment of sleep was limited to baseline to follow-up changes in pain interference with sleep. Although this is a recommended outcome measures in pain therapy trials, ${ }^{80}$ other outcomes such as validated sleep instruments ${ }^{81,82}$ and a global sleep impression may have yielded different results. Fourth, actigraphic sleep data were available for a subset of participants. However, since the same proportion of participants in the two groups contributed, it is doubtful that group differences resulted from selection bias. Finally, the study results were obtained using a TENS device that utilized a specific electrode configuration and stimulation parameters, and therefore the conclusions may not generalize to other TENS instruments and methods.

\section{Conclusion}

Regular TENS use improved subjective and objective sleep measures in individuals with CLBP. About half of the study participants exhibited improved ( $\geq 1$ point) pain interference with sleep. When compared with the unimproved group, the improved group had longer total sleep time and fewer PLMs measured by actigraphy. These results suggest that one of the ways in which TENS may improve CLBP is through improvement in sleep. Future studies evaluating the clinical effectiveness of TENS in CLBP should consider sleep in addition to pain intensity.

\section{Data sharing statement}

The study authors will make the study data available to researchers interested in its use for academic, non-commercial purposes.

\section{Acknowledgment}

This study was funded by NeuroMetrix, Inc.

\section{Author contributions}

SNG was responsible for designing the study, conducting statistical analyses, and initial preparation of the article. TCF designed and MM implemented the actigraphy algorithms used in the TENS device. XK was responsible for extracting data from the online database and assisting in the statistical analyses. All authors contributed to the data analyses, drafting and revisions of the paper, gave final approval of the version to be published, and agree to be accountable for all aspects of the work.

\section{Disclosure}

SNG, TCF, MM, and XK are employees and shareholders of NeuroMetrix, Inc. The authors report no other conflicts of interest in this work.

\section{References}

1. Vance CG, Dailey DL, Rakel BA, Sluka KA. Using TENS for pain control: the state of the evidence. Pain Manag. 2014;4(3):197-209.

2. Fox EJ, Melzack R. Transcutaneous electrical stimulation and acupuncture: comparison of treatment for low-back pain. Pain. 1976;2(2):141-148.

3. Resende L, Merriwether E, Rampazo ÉP, et al. Meta-analysis of transcutaneous electrical nerve stimulation for relief of spinal pain. Eur $J$ Pain. 2018;22(4):663-678.

4. Jauregui JJ, Cherian JJ, Gwam CU, et al. A meta-analysis of transcutaneous electrical nerve stimulation for chronic low back pain. Surg Technol Int. 2016;28:296-302. 
5. Dubinsky RM, Miyasaki J. Assessment: efficacy of transcutaneous electric nerve stimulation in the treatment of pain in neurologic disorders (an evidence-based review): report of the therapeutics and technology assessment subcommittee of the American Academy of Neurology. Neurology. 2010;74(2):173-176.

6. Brosseau L, Milne S, Robinson V, et al. Efficacy of the transcutaneous electrical nerve stimulation for the treatment of chronic low back pain. Spine (Phila Pa 1976). 2002;27(6):596-603.

7. Khadilkar A, Milne S, Brosseau L, et al. Transcutaneous electrical nerve stimulation (TENS) for chronic low-back pain. Cochrane Database Syst Rev. 2005;3:CD003008.

8. Wu LC, Weng PW, Chen CH, et al. And meta-analysis of transcutaneous electrical nerve stimulation in treating chronic back pain. Reg Anesth Pain Med. 2018;43(4):425-433.

9. Moran F, Leonard T, Hawthorne S, et al. Hypoalgesia in response to transcutaneous electrical nerve stimulation (TENS) depends on stimulation intensity. J Pain. 2011;12(8):929-935.

10. Bennett MI, Hughes N, Johnson MI. Methodological quality in randomised controlled trials of transcutaneous electric nerve stimulation for pain: low fidelity may explain negative findings. Pain. 2011;152(6):1226-1232.

11. Pallett EJ, Rentowl P, Johnson MI, Watson PJ. Implementation fidelity of self-administered transcutaneous electrical nerve stimulation (TENS) in patients with chronic back pain. Clin J Pain. 2014;30(3):224-231.

12. Gladwell PW, Badlan K, Cramp F, Palmer S. Direct and indirect benefits reported by users of transcutaneous electrical nerve stimulation for chronic musculoskeletal pain: qualitative exploration using patient interviews. Phys Ther. 2015;95(11):1518-1528.

13. Atkinson JH, Ancoli-Israel S, Slater MA, Garfin SR, Gillin C. Subjective sleep disturbance in chronic back pain. Clin J Pain. 1988;4(4):225-232.

14. Marty M, Rozenberg S, Duplan B, et al. Quality of sleep in patients with chronic low back pain: a case-control study. Eur Spine J. 2008;17(6):839-844

15. Alsaadi SM, McAuley JH, Hush JM, Maher CG. Prevalence of sleep disturbance in patients with low back pain. Eur Spine J. 2011;20(5):737-743.

16. O’Donoghue GM, Fox N, Heneghan C, Hurley DA. Objective and subjective assessment of sleep in chronic low back pain patients compared with healthy age and gender matched controls: a pilot study. $B M C$ Musculoskelet Disord. 2009;10(1):122.

17. Kelly GA, Blake C, Power CK, O’Keeffe D, Fullen BM. The association between chronic low back pain and sleep: a systematic review. Clin J Pain. 2011;27(2):169-181.

18. van de Water AT, Eadie J, Hurley DA. Investigation of sleep disturbance in chronic low back pain: an age- and gender-matched case-control study over a 7-night period. Man Ther. 2011;16(6):550-556.

19. Casarett D, Karlawish J, Sankar P, Hirschman K, Asch DA. Designing pain research from the patient's perspective: what trial end points are important to patients with chronic pain? Pain Med. 2001;2(4):309-316.

20. Turk DC, Dworkin RH, Revicki D, et al. Identifying important outcome domains for chronic pain clinical trials: an IMMPACT survey of people with pain. Pain. 2008;137(2):276-285.

21. Gozani SN. Fixed-site high-frequency transcutaneous electrical nerve stimulation for treatment of chronic low back and lower extremity pain. J Pain Res. 2016;9:469-479.

22. Kong X, Gozani SN. Effectiveness of fixed-site high-frequency transcutaneous electrical nerve stimulation in chronic pain: a large-scale, observational study. J Pain Res. 2018;11:703-714.

23. Gozani SN, Kong X. Real-world evidence for the widespread effects of fixed-site high-frequency transcutaneous electrical nerve stimulation in chronic pain. J Pain Relief. 2018;7(4).

24. Vinik A, Emir B, Parsons B, Cheung R. Prediction of pregabalinmediated pain response by severity of sleep disturbance in patients with painful diabetic neuropathy and post-herpetic neuralgia. Pain Med. 2014;15(4):661-670.

25. Latremoliere A, Woolf CJ. Central sensitization: a generator of pain hypersensitivity by central neural plasticity. J Pain. 2009;10(9):895-926.
26. von Elm E, Altman DG, Egger M, et al. The strengthening the reporting of observational studies in epidemiology (STROBE) statement: guidelines for reporting observational studies. PLoS Med. 2007;4(10): e296.

27. Johnson MI. Transcutaneous Electrical Nerve Stimulation (TENS): Research to Support Clinical Practice. Oxford: Oxford University Press; 2014.

28. Dailey DL, Rakel BA, Vance CG, et al. Transcutaneous electrical nerve stimulation reduces pain, fatigue and hyperalgesia while restoring central inhibition in primary fibromyalgia. Pain. 2013;154(11):2554-2562.

29. Rao VR, Wolf SL, Gersh MR. Examination of electrode placements and stimulating parameters in treating chronic pain with conventional transcutaneous electrical nerve stimulation (TENS). Pain . 1981;11(1):37-47.

30. Neto MLP, Maciel LYS, Cruz KML, Filho VJS, Bonjardim LR, Desantana JM. Does electrode placement influence tens-induced antihyperalgesia in experimental inflammatory pain model? Braz J Phys Ther. 2017;21(2):92-99.

31. Pantaleão MA, Laurino MF, Gallego NL, et al. Adjusting pulse amplitude during transcutaneous electrical nerve stimulation (TENS) application produces greater hypoalgesia. J Pain. 2011;12(5): 581-590.

32. Marchand S, Charest J, Li J, Chenard JR, Lavignolle B, Laurencelle L. Is TENS purely a placebo effect? A controlled study on chronic low back pain. Pain. 1993;54(1):99-106.

33. Ancoli-Israel S, Cole R, Alessi C, Chambers M, Moorcroft W, Pollak $\mathrm{CP}$. The role of actigraphy in the study of sleep and circadian rhythms. Sleep. 2003;26(3):342-392.

34. Sadeh A. The role and validity of actigraphy in sleep medicine: an update. Sleep Med Rev. 2011;15(4):259-267.

35. Tryon WW. Issues of validity in actigraphic sleep assessment. Sleep. 2004;27(1):158-165.

36. Lichstein KL, Stone KC, Donaldson J, et al. Actigraphy validation with insomnia. Sleep. 2006;29(2):232-239.

37. Ohayon M, Wickwire EM, Hirshkowitz M, et al. National Sleep Foundation's sleep quality recommendations: first report. Sleep Health. 2017;3(1):6-19.

38. Hirshkowitz M, Whiton K, Albert SM, et al. National Sleep Foundation's updated sleep duration recommendations: final report. Sleep Health. 2015;1(4):233-243.

39. Natarajan R. Review of periodic limb movement and restless leg syndrome. J Postgrad Med. 2010;56(2):157-162.

40. Gschliesser V, Frauscher B, Brandauer E, et al. PLM detection by actigraphy compared to polysomnography: a validation and comparison of two actigraphs. Sleep Med. 2009;10(3):306-311.

41. Sforza E, Johannes M, Claudio B. The PAM-RL ambulatory device for detection of periodic leg movements: a validation study. Sleep Med. 2005;6(5):407-413.

42. Montplaisir J, Boucher S, Poirier G, Lavigne G, Lapierre O, Lespérance P. Clinical, polysomnographic, and genetic characteristics of restless legs syndrome: a study of 133 patients diagnosed with new standard criteria. Mov Disord. 1997;12(1):61-65.

43. Okura K, Lavigne GJ, Huynh N, Manzini C, Fillipini D, Montplaisir JY. Comparison of sleep variables between chronic widespread musculoskeletal pain, insomnia, periodic leg movements syndrome and control subjects in a clinical sleep medicine practice. Sleep Med. 2008;9(4):352-361.

44. Pennestri MH, Montplaisir J, Fradette L, Lavigne G, Colombo R, Lanfranchi PA. Blood pressure changes associated with periodic leg movements during sleep in healthy subjects. Sleep Med. 2013;14(6):555-561.

45. Oksenberg A, Gadoth N. Periodic limb movements and heart rate changes. J Clin Sleep Med. 2012;8(4):447-449.

46. Karadeniz D, Ondze B, Besset A, Billiard M. EEG arousals and awakenings in relation with periodic leg movements during sleep. J Sleep Res. 2000;9(3):273-277.

47. Karadeniz D, Ondze B, Besset A, Billiard M. Are periodic leg movements during sleep (PLMS) responsible for sleep disruption in insomnia patients? Eur J Neurol. 2000;7(3):331-336. 
48. Hornyak M, Riemann D, Voderholzer U. Do periodic leg movements influence patients' perception of sleep quality? Sleep Med. 2004;5(6):597-600.

49. Ferri R, Fulda S, Allen RP, et al. World association of sleep medicine (WASM) 2016 standards for recording and scoring leg movements in polysomnograms developed by a joint task force from the International and the European Restless Legs Syndrome Study Groups (IRLSSG and EURLSSG). Sleep Med. 2016;26:86-95.

50. Ferree T, Gozani S, Winkelman J. Pilot study of sleep/wake classification by leg-worn actigraphy (P1.113). Neurology. 2018;90(15 Suppl).

51. Ferree T, Gozani S, Winkelman J. 0677 Pilot study of periodic leg movement classification with leg-worn actigraphy. Sleep. 2018;41(suppl_1):A251.

52. Berger AM, Wielgus KK, Young-McCaughan S, Fischer P, Farr L, Lee KA. Methodological challenges when using actigraphy in research. J Pain Symptom Manage. 2008;36(2):191-199.

53. Cleeland CS, Ryan KM. Pain assessment: global use of the brief pain inventory. Ann Acad Med Singapore. 1994;23(2):129-138.

54. Dworkin RH, Turk DC, Wyrwich KW, et al. Interpreting the clinical importance of treatment outcomes in chronic pain clinical trials: IMMPACT recommendations. J Pain. 2008;9(2):105-121.

55. Scherer M, Hansen H, Gensichen J, et al. Association between multimorbidity patterns and chronic pain in elderly primary care patients: a cross-sectional observational study. BMC Fam Pract. 2016;17(1):68.

56. Edwards RR, Almeida DM, Klick B, Haythornthwaite JA, Smith MT. Duration of sleep contributes to next-day pain report in the general population. Pain. 2008;137(1):202-207.

57. Nitter AK, Pripp AH, Forseth KØ. Are sleep problems and non-specific health complaints risk factors for chronic pain? A prospective population-based study with 17 year follow-up. Scand J Pain. 2012;3(4): 210-217.

58. Högl B. Periodic limb movements are associated with disturbed sleep. Pro. J Clin Sleep Med. 2007;3(1):12-14.

59. Johnson MI, Ashton CH, Thompson JW. An in-depth study of long-term users of transcutaneous electrical nerve stimulation (TENS). Implications for clinical use of TENS. Pain. 1991;44(3):221-229.

60. Fishbain DA, Chabal C, Abbott A, Heine LW, Cutler R. Transcutaneous electrical nerve stimulation (TENS) treatment outcome in long-term users. Clin J Pain. 1996;12(3):201-214.

61. Wilson KG, Watson ST, Currie SR. Daily diary and ambulatory activity monitoring of sleep in patients with insomnia associated with chronic musculoskeletal pain. Pain. 1998;75(1):75-84.

62. Finan PH, Goodin BR, Smith MT. The association of sleep and pain: an update and a path forward. J Pain. 2013;14(12):1539-1552.

63. Smith MT, Haythornthwaite JA. How do sleep disturbance and chronic pain inter-relate? Insights from the longitudinal and cognitive-behavioral clinical trials literature. Sleep Med Rev. 2004;8(2):119-132.

64. DeSantana JM, Walsh DM, Vance C, Rakel BA, Sluka KA. Effectiveness of transcutaneous electrical nerve stimulation for treatment of hyperalgesia and pain. Curr Rheumatol Rep. 2008;10(6):492-499.

65. Bara-Jimenez W, Aksu M, Graham B, Sato S, Hallett M. Periodic limb movements in sleep: state-dependent excitability of the spinal flexor reflex. Neurology. 2000;54(8):1609-1616.
66. Skljarevski V, Ramadan NM. The nociceptive flexion reflex in humans - review article. Pain. 2002;96(1):3-8.

67. Sandrini G, Serrao M, Rossi P, Romaniello A, Cruccu G, Willer JC. The lower limb flexion reflex in humans. Prog Neurobiol. 2005;77(6): 353-395.

68. Tsang HH. Diffuse Inhibition of Flexion Reflex by Transcutaneous Electrical Nerve Stimulation (Tens) in Man. [theses]. Montreal: McGill University; 1986

69. Chan CWY, Tsang H. Inhibition of the human flexion reflex by low intensity, high frequency transcutaneous electrical nerve stimulation (TENS) has a gradual onset and offset. Pain. 1987;28(2):239-253.

70. Levin MF, Hui-Chan CW. Relief of hemiparetic spasticity by TENS is associated with improvement in reflex and voluntary motor functions. Electroencephalogr Clin Neurophysiol. 1992;85(2):131-142.

71. Aydin G, Tomruk S, Keleş I, Demir SO, Orkun S. Transcutaneous electrical nerve stimulation versus baclofen in spasticity: clinical and electrophysiologic comparison. Am J Phys Med Rehabil. 2005;84(8): 584-592.

72. Karakoyun A, Boyraz Ismail, Gunduz R, Karamercan A, Ozgirgin N. Electrophysiological and clinical evaluation of the effects of transcutaneous electrical nerve stimulation on the spasticity in the hemiplegic stroke patients. J Phys Ther Sci. 2015;27(11):3407-3411.

73. Winkelman JW, La M, Platt S, Schoerning L. Pilot open-label trial of transcutaneous electrical nerve stimulation (TENS) below the knee for the treatment of restless legs syndrome (RLS). Sleep. 2016;39(Abstract Supplement):A234.

74. van Dijk KR, Luijpen MW, van Someren EJ, Sergeant JA, Scheltens P, Scherder EJ. Peripheral electrical nerve stimulation and rest-activity rhythm in Alzheimer's disease. J Sleep Res. 2006;15(4):415-423.

75. Neikrug AB, Donaldson G, Iacob E, Williams SL, Hamilton CA, Okifuji A. Activity rhythms and clinical correlates in fibromyalgia. Pain. 2017;158(8):1417-1429.

76. Kadono M, Nakanishi N, Yamazaki M, Hasegawa G, Nakamura N, Fukui M. Various patterns of disrupted daily rest-activity rhythmicity associated with diabetes. J Sleep Res. 2016;25(4):426-437.

77. Spira AP, Runko VT, Finan PH, et al. Circadian rest/activity rhythms in knee osteoarthritis with insomnia: a study of osteoarthritis patients and pain-free controls with insomnia or normal sleep. Chronobiol Int. 2015;32(2):242-247.

78. Huang Z, Chen J, Ma J, Pei F, Kraus V. Based on meta-analysis, placebo responses are less inflated for objective compared to subjective measures in osteoarthritis trials. Osteoarthritis Cartilage. 2018;26:S346-S347.

79. Meissner K, Distel H, Mitzdorf U. Evidence for placebo effects on physical but not on biochemical outcome parameters: a review of clinical trials. BMC Med. 2007;5(1):3.

80. Dworkin RH, Turk DC, Farrar JT, et al. Core outcome measures for chronic pain clinical trials: IMMPACT recommendations. Pain. 2005;113(1-2):9-19.

81. Buysse DJ, Reynolds CF, 3rd, Monk TH, Berman SR, Kupfer DJ. The Pittsburgh sleep quality index: a new instrument for psychiatric practice and research. Psychiatry Res. 1989;28(2):193-213.

82. Johns MW. A new method for measuring daytime sleepiness: the Epworth Sleepiness Scale. Sleep. 1991;14(6):540-545.
Journal of Pain Research

\section{Publish your work in this journal}

The Journal of Pain Research is an international, peer reviewed, open access, online journal that welcomes laboratory and clinical findings in the fields of pain research and the prevention and management of pain. Original research, reviews, symposium reports, hypothesis formation and commentaries are all considered for publication.

\section{Dovepress}

The manuscript management system is completely online and includes a very quick and fair peer-review system, which is all easy to use. Visit http://www.dovepress.com/testimonials.php to read real quotes from published authors. 